\title{
Entrapment of Device
}

National Cancer Institute

\section{Source}

National Cancer Institute. Entrapment of Device. NCI Thesaurus. Code C63210.

Issue associated with the device and/or device accessories caught within patient vasculature, tissue, or other device. 